\title{
Burden of Disease in Chronic Rhinosinusitis with Nasal Polyps
}

This article was published in the following Dove Press journal:

Journal of Asthma and Allergy

\author{
Claus Bachert ${ }^{1,2}$ \\ Neil Bhattacharyya ${ }^{3}$ \\ Martin Desrosiers (1D ${ }^{4}$ \\ Asif $\mathrm{H}$ Khan ${ }^{1,5}$ \\ 'Upper Airways Research Laboratory, \\ Department of Otorhinolaryngology, \\ Ghent University, Ghent, Belgium; \\ ${ }^{2}$ CLINTEC, Karolinska Institutet, \\ Stockholm, Sweden; ${ }^{3}$ Department of \\ Otolaryngology, Harvard Medical School, \\ Boston, MA, USA; ${ }^{4}$ Centre de recherche \\ du Centre hospitalier de l'Université de \\ Montréal (CRCHUM), Montreal, QC, \\ Canada; ${ }^{5}$ Sanofi, Chilly-Mazarin, France
}

Correspondence: Claus Bachert

Upper Airways Research Laboratory,

Ghent University, C. Heymanslaan I0,

Ghent, B-9000, Belgium

Tel +32 93326880

Email Claus.Bachert@UGent.be

\begin{abstract}
Chronic rhinosinusitis with nasal polyps (CRSwNP) is a predominantly type 2 inflammation-mediated disease of the nasal mucosa and paranasal sinuses with an underrecognized clinical, humanistic, and economic burden. Patients with CRSwNP experience a high symptom burden, including nasal congestion, loss of smell, and rhinorrhea, which has a negative impact on physical and mental health-related quality of life, including sleep quality. Existing medical and surgical interventions, including local and systemic corticosteroids and endoscopic sinus surgery, may be associated with recurrence of nasal polyps and associated symptoms and with an increased risk of short- and long-term adverse effects, especially with repeated or long-term use. Because type 2 inflammation is implicated in the pathogenesis of several coexisting diseases, patients with CRSwNP often have comorbid asthma and/or nonsteroidal anti-inflammatory drug-exacerbated respiratory disease. These patients, as well as those with high corticosteroid use and/or sinonasal surgical history, have more severe disease and associated symptom burden and represent a difficult-to-treat population under the existing management paradigm. This article reviews the clinical, humanistic, and economic burden of CRSwNP; it highlights the unmet need for effective and safe CRSwNP therapies that effectively control symptoms and minimize recurrence by targeting the underlying type 2 inflammatory disease pathophysiology.
\end{abstract}

Keywords: disease severity, healthcare economics, paranasal sinus disease, quality of life

\section{Introduction}

Chronic rhinosinusitis with nasal polyps (CRSwNP) is a chronic inflammatory disease of the nasal mucosa and paranasal sinuses that is associated with significant morbidity and reduced health-related quality of life (HRQoL). ${ }^{1-3}$ The pathophysiology of CRSwNP is associated with local (sinonasal) and systemic (lower airway) inflammation, with persistent symptoms of nasal congestion, rhinorrhea, and loss of smell that have a significant impact on HRQoL. ${ }^{4}$ Most patients with CRSwNP show evidence of type 2 airway inflammation, and these patients have the highest disease burden. ${ }^{5-8}$ As a result of the shared type 2 inflammatory pathway implicated in several coexisting diseases, patients with CRSwNP often have comorbid asthma and/or nonsteroidal antiinflammatory drug-exacerbated respiratory disease (NSAID-ERD).

The disease burden with significantly lower physical and mental HRQoL than population norms is particularly high in CRSwNP. This is particularly in patients with comorbid asthma and/or NSAID-ERD 9 and in patients who need repeated treatment with corticosteroids and/or sinonasal surgeries to alleviate its uncontrolled symptoms. The impact on HRQoL is comparable with other chronic diseases such as chronic obstructive pulmonary disease (COPD), asthma, and diabetes. ${ }^{10,11}$ 
CRSwNP can be difficult to treat in patients with the highest burden. The current treatment paradigm involves corticosteroids and/or sinonasal surgery, but these options may be associated with recurrence of nasal polyps, and patients may require revision surgery. Additionally, there exist non-negligible risks associated with both repeated steroid use and surgery. ${ }^{12,13}$ A qualitative study reported that patients can become frustrated with the management of their disease and often feel that the impact on their quality of life is not fully recognized. ${ }^{14}$

For this review, a targeted search of the literature was performed to examine the burden of disease for CRSwNP. Although previous literature reviews have focused on specific aspects of unmet need, this review evaluates a broader set of outcomes, with a focus on burden: the clinical, humanistic, and economic aspects of the disease, and the impact on HRQoL of the patients. We searched MEDLINE (via PubMed) and Embase for articles using search terms including "nasal polyposis," "rhinosinusitis," "chronic rhinosinusitis," and "chronic rhinosinusitis with nasal polyps," and articles were chosen for inclusion based on their relevance to the topic. We also referenced expert position papers. In this targeted literature review, we highlight the epidemiology and pathology of CRSwNP, examine the clinical, humanistic, and economic burden of the disease, and close with a review of the current treatment paradigm and its limitations.

\section{Epidemiology and Pathology}

The proportion of adults in the USA ${ }^{15}$ who would meet the symptom criteria of chronic rhinosinusitis (CRS) is estimated to be $2.1 \%$ and, based on population studies, it is estimated to be between $2.1 \%$ and $4.3 \%$ in Europe. ${ }^{16,17}$ Among all patients with CRS, approximately 20-30\% have CRSwNP., ${ }^{3,18}$ Overall, given the requirement for nasal endoscopy to make a reliable diagnosis, prevalence estimates for nasal polyps based on questionnaire data may not represent an accurate estimate. ${ }^{19}$

CRSwNP is typically a disease of middle age, with a peak incidence between the fourth and fifth decades of life. ${ }^{1}$ Despite the presence of nasal symptoms, which develop slowly, many patients with CRSwNP do not seek medical attention for their sinonasal symptoms. ${ }^{17}$ Thus, many patients diagnosed in middle age may have severe disease in spite of experiencing symptoms for decades; among those who are diagnosed at a younger age, there is the expectation of a long-term burden of illness on the patient. Although females are less likely to be affected than males, females are more likely than males to experience greater severity of symptoms. ${ }^{3}$

The etiology of CRSwNP is yet to be fully elucidated. The presence of persistent symptoms such as nasal congestion, loss of smell, and rhinorrhea for $>3$ months is the basis for diagnosing CRSwNP. ${ }^{20}$ Postnasal drip is also common, whereas facial congestion and pain are variable. ${ }^{20,21}$ It should be noted that severe symptoms do not occur at the same intensity throughout the disease severity spectrum.

CRS is not a homogeneous disease but rather a heterogeneous disorder with multiple inflammatory endotypes. ${ }^{22}$ Affected patients have bilateral benign edematous polyps extending from the paranasal sinuses to the nasal cavity, with increased levels of cytokines and mediators $^{23}$ and an intense inflammatory infiltrate as a typical finding. ${ }^{1}$ In Western countries, CRSwNP is most often associated with eosinophilic inflammation whose characteristics include elevated levels of immunoglobulin E (IgE), interleukin (IL)-4, and IL-5 compared with patients without nasal polyps. ${ }^{22,24,25}$ The polyclonal and preferential activation of $\mathrm{T}$ helper type 2 cells, a feature of this inflammatory pattern, is implicated in the amplification of mucosal inflammation ${ }^{26}$ and is typical of patients with more severe CRSwNP. Although of a predominantly eosinophilic phenotype, with a less common neutrophilic inflammatory subtype observed, no specific genetic or environmental factors are strongly associated with the disorder and defects in the sinonasal epithelial barrier; bacterial exposure and dysregulation of the immune system may all play a role. ${ }^{1,3,24}$ Patients with allergic fungal rhinosinusitis, characterized by elevated antifungal $\operatorname{IgE}$ and eosinophilic mucus, represent a unique subgroup presenting with unilateral CRSwNP. ${ }^{27}$

CRSwNP symptoms are associated with local and systemic inflammation. ${ }^{4}$ Localized inflammation is characterized by a dysfunctional interaction between the sinonasal mucosa and the environment. CRSwNP is associated with higher rates of colonization with Staphylococcus aureus in the upper airway leading to biofilm formation, immune dysregulation, inflammation, and barrier dysfunction, contributing to recalcitrant disease. ${ }^{23,28,29}$ Systemic immunologic cross-talk may play a role, with symptoms of CRS resulting from simultaneous irritation of the upper and lower airways. ${ }^{4}$ There is also a suggestion that reflexes mediated via the nervous system, possibly via the nodose ganglion/vagus nerve, may play a role as occurs in asthma. $^{30}$ 


\section{Clinical Burden}

The clinical presentation of CRSwNP may include nasal congestion, rhinorrhea/postnasal drip, facial pain or headache, impaired sense of smell, and sleep disturbance or fatigue; uncontrolled disease is defined as the persistence of these symptoms on most days of the week and the need for rescue treatment. ${ }^{20}$ The clinical burden of CRSwNP can be greatly impacted by the presence of comorbid disease.

Bilateral eosinophilic CRSwNP is often associated with non-allergic asthma and intolerance to NSAIDs; NSAID-ERD represents a serious and recurrent clinical form of the disease with pronounced disease burden based on objective measures. ${ }^{1,31}$ In patients with CRSwNP, up to $65 \%$ and $26 \%$ have comorbid asthma and NSAID-ERD, respectively. ${ }^{1,26,32-34}$ Among patients with CRSwNP and comorbid asthma or NSAID-ERD, increased disease severity associated with type 2 inflammation is additionally characterized by recurrence of nasal polyps, higher rates of revision surgery, systemic corticosteroid (SCS) dependence, and poor asthma control. ${ }^{35,36}$

In patients with CRSwNP, the prevalence of comorbid asthma is reported to be up to $65 \%,{ }^{22,32,37}$ much higher than the prevalence of asthma in the general US population, which is $8.5 \%{ }^{38}$ In a recent study involving patients with severe asthma, $43 \%$ also suffered from CRSwNP. ${ }^{39}$ CRSwNP has been associated with adult-onset asthma (onset after 12 years of age) or late-onset asthma (onset after 40 years of age)..$^{40}$ The prevalence of CRSwNP and that of comorbid asthma increased with age and was particularly evident in those aged $\geq 40$ years. ${ }^{41}$ Patients with late-onset asthma were less frequently atopic and more likely to have CRSwNP. ${ }^{39}$

NSAID-ERD is one of the most serious, recurrent, and treatment-resistant comorbidities associated with CRSwNP, and it is often found in association with other type 2 inflammatory diseases such as asthma. ${ }^{1,26,31}$ NSAID-ERD in particular is thought to place an especially high clinical burden on affected patients. ${ }^{9,34}$ The frequent recurrence of nasal polyps after surgery and a requirement for high-dose SCS to manage asthma, if present, reflect the persistent and aggressive nature of the disease. ${ }^{42}$

In the USA, it is estimated that patients with CRSwNP with NSAID-ERD undergo an average of three sinus operations during their lifetime. ${ }^{33}$ In addition to undergoing more operations, those with NSAID-ERD were also younger at the time of their first surgery compared with patients with CRSwNP without NSAID-ERD. One study of patients with
CRS undergoing revision sinus surgery found that the presence of nasal polyps was associated with the presence of comorbid aspirin sensitivity, with additional evidence of disease burden in terms of higher endoscopy scores, computed tomography (CT) scores, and absolute eosinophil counts. ${ }^{31}$ Thus, NSAID-ERD is a difficult-to-treat disease both from a pharmacologic and a surgical perspective. ${ }^{43}$

\section{Humanistic Burden}

From a patient perspective, CRSwNP carries a substantial burden that has a significant impact on HRQoL. Compared with those without CRSwNP, patients with CRSwNP experience higher symptom scores and greater severity of clinical disease. ${ }^{22,25,34}$ The presence of CRSwNP is not only associated with greater burden of disease at presentation but also with worse disease severity despite sinonasal surgery. ${ }^{31}$

Patients with CRSwNP suffer in their overall health in a way that cannot be described by disease-specific measures alone. The impact of CRSwNP on overall HRQoL has been reported to be comparable with other chronic diseases such as COPD, asthma, and diabetes. ${ }^{10,11}$ In a study comparing health state utility values, CRS demonstrated impairment on a par with end-stage renal disease, moderate asthma, Parkinson's disease, and coronary artery disease. ${ }^{44}$ A study using the 36-Item Short-Form Health Survey (SF-36) questionnaire reported that patients with CRS had lower overall scores compared with population norms, and they had significantly worse bodily pain and social functioning scores than those with congestive heart failure, COPD, angina, or sciatica. ${ }^{45}$ Another study, also utilizing SF-36, reported that patients with CRSwNP had worse physical and mental health compared with population norms. ${ }^{9}$

CRSwNP and asthma are individually associated with substantial disease burden, and patients with CRSwNP and comorbid asthma have an even greater combined burden that has a serious impact on HRQoL. ${ }^{9,46}$ The presence of asthma resulted in significantly higher symptom scores compared with patients without asthma. ${ }^{25}$ Compared with non-asthmatic controls, patients with CRSwNP scored significantly lower in the Mini Asthma Quality of Life Questionnaire, irrespective of asthma. ${ }^{32}$ In one study, poor asthma control correlated with high sinus CT scores in CRSwNP. ${ }^{47}$

CRSwNP is frequently associated with severe olfactory loss. One of the most troublesome and recalcitrant symptoms in patients with CRSwNP, loss of smell, correlates 
with disease severity and has a substantial impact on HRQoL. ${ }^{48}$ A Korean study that assessed HRQoL and psychological symptoms in patients with CRSwNP found that olfactory dysfunction may also have significant effects on psychological health, including higher levels of depression, anxiety, and phobia. ${ }^{48}$ A qualitative study on olfactory loss found that olfactory impairment exposes individuals to potential environmental dangers such as the inability to identify expired foods or to detect smoke or gas, reduces enjoyment of food, and can interfere with the evocation of memories. ${ }^{49}$ CRSwNP not only has a major impact on general and disease-specific HRQoL but also impairs sleep quality and nasal patency, and increases daytime sleepiness and the risk of sleep apnea, ${ }^{50}$ all of which may negatively affect patient mental health.

A qualitative study that evaluated patient experiences of CRS found that many patients are self-conscious about their condition due to the impacts of constant nasal congestion and/or discharge; other patients experienced depression. Loss of confidence was common due to the inability to speak clearly in social and work situations; poor or disrupted sleep occurred frequently resulting in problems with fatigue and ability to concentrate; loss of smell and taste reduced enjoyment in socializing and eating out; participants described forgetting what it was like to be normal; many participants reported experiencing frustrations with the primary care management of their symptoms; and many participants reported that health care professionals failed to recognize the impact on HRQoL. ${ }^{14}$

\section{Economic Burden}

CRSwNP has significant direct and indirect costs to patients and society. Among patients with CRSwNP and comorbid asthma or NSAID-ERD, increased disease severity associated with type 2 inflammation is additionally characterized by higher costs and health care utilization. ${ }^{35}$ NSAID-ERD, in particular, is thought to place an especially high financial burden on affected patients. ${ }^{34}$ In the USA, total annual health care costs among patients with CRSwNP were $\$ 11,507$, significantly higher than the reference population, leading to a total cost of $\$ 5.7$ billion. ${ }^{35} \mathrm{CRSwNP}$ also has indirect and productivity-related costs. Costs to the patient relating to absenteeism, presenteeism, and lost work productivity are likely to be substantial, with CRS associated with an average of 4.8 days of missed work per year. ${ }^{4}$ Indeed, the mean annual indirect costs for patients with inadequately controlled CRSwNP have been estimated at $\$ 7182$ per patient, ${ }^{51}$ indicating that the total cost of CRSwNP per year exceeds $\$ 15,000$. In a recent study in Europe, direct costs for patients with CRSwNP were $€ 1501$ per patient/year, with indirect costs of $€ 5659$ per patient/year, largely due to outpatient/hospital visits and productivity loss, respectively. ${ }^{52}$

A health economic analysis of SCS use in patients with CRSwNP that considered the impact of adverse events found that the breakeven threshold for which surgery was favored over medical therapy occurred when $>1$ SCS course was given every 2 years. ${ }^{53}$ The threshold number of SCS courses per year at which risks of medical therapy exceed risks of surgery were 0.21 for CRSwNP alone, 0.55 for CRSwNP with asthma, and 1.82 for CRSwNP with asthma and NSAID-ERD. ${ }^{13}$ Analyses of this nature provide evidence-based thresholds for clinicians to utilize when making clinical decisions with patients on the relative utility of surgery versus repeated corticosteroid use.

\section{Current Treatment Paradigm and Its Limitations}

Evidence-based recommendations for the management of CRSwNP call for the use of intranasal and oral corticosteroids/SCS where the goal of treatment is to achieve and maintain clinical disease control and avoid complications. $^{4,20}$ Disease control is defined as the absence of symptoms or symptoms no longer being bothersome, together with a healthy mucosa and need for topical medication alone. ${ }^{4}$ When suboptimally controlled, CRSwNP worsens the course of lower airway disease. ${ }^{53}$

There is good evidence that both intranasal corticosteroids and SCS are effective to some extent in the management of CRSwNP. However, there are also limitations with these treatments, and their short-lived benefits need to be balanced with the need for long-term control and safety. ${ }^{13,53}$ Existing data support the infrequent use of SCS in the immediate- and short-term periods for patients with CRSwNP; however, their long-term benefits are limited. ${ }^{53}$ SCS treatment can lead to serious treatmentrelated adverse effects, and even short-term corticosteroid use is associated with an increased risk of acute complications such as sepsis, venous thromboembolism, and fracture. ${ }^{54,55}$ 
If CRSwNP is not controlled in a patient after a period of time, a CT scan and surgery may be considered. ${ }^{4}$ While endoscopic surgery has greatly improved the safety of sinonasal surgery, significant risks remain, with minor complications reported in $5 \%$ of routine endoscopic interventions and major complications reported in $0.5-1 \%$ of them. ${ }^{12}$ The long-term revision rates for endoscopic sinus surgery for CRS exceed $15 \%$, with the presence of nasal polyps having the largest impact on the risk of revision surgery with success rates reported of around $50-70 \%{ }^{20,56}$

The current treatment paradigm for the management of CRSwNP has significant limitations. The recurrence rate of CRSwNP after surgery ranges from $20 \%$ to $60 \%$ within 18 months to 4 years follow-up ${ }^{35}$ to $79 \%$ over a 12 -year period. ${ }^{57}$ Recurrences are particularly common in patients with more severe disease such as that associated with comorbid asthma or who have undergone prior surgeries. $^{20,53}$ Among patients with CRSwNP alone, CRSwNP with asthma, and CRSwNP with asthma and NSAID-ERD, median times to revision surgery were 20, 11, and 7 years, respectively. ${ }^{13}$ Median times to polyp recurrence were 20,4 , and 0.66 years, respectively. Intense eosinophilia together with asthma and NSAIDERD are associated with a 4.5 -fold increased risk of recurrence following initial surgery. ${ }^{1}$

Many patients with prior SCS use or surgery remain symptomatic. Among patients who undergo endoscopic sinus surgery for CRSwNP, failure rates, defined as persistent symptoms for $>6$ months after surgery, are high, with one study reporting $23 \%$ of patients with persistent symptoms after surgery. ${ }^{53}$ In a qualitative study of patient perspective on revision surgery, patients who had undergone more surgeries were more pessimistic in their expectations and objectives for surgical treatment (Saydy, unpublished). In a qualitative study evaluating patient experiences of CRS, patients often described sinus surgery as a temporary solution, with the expectation that surgery may not fully resolve symptoms or that symptoms would return and further surgery may be required. ${ }^{14}$ Indeed, the data support this, as $59 \%$ of the European GALEN cohort of patients with CRSwNP required revision surgery and $7 \%$ of patients had undergone $\geq 4$ surgical procedures. ${ }^{40}$

Patients with persistent CRSwNP symptoms despite appropriate medical and/or surgical management are defined as difficult to treat. ${ }^{4}$ Due to the chronic and recurring nature of CRSwNP, even after surgery and existing medical approaches that focus on non-specific reduction of local inflammation (and lower airway inflammation in the case of SCS), there is a medical need for targeted treatment of nasal polyps and the underlying mechanisms of chronic sinus inflammation. Despite available therapies, the burden of CRSwNP in adults is substantial and demonstrates an unmet need for effective management strategies. Novel, well-tolerated therapies that provide effective symptom control and minimize recurrence rates of nasal polyps are needed. A number of biologic therapies targeting type 2 inflammation have been investigated in CRSwNP. ${ }^{5,58-62}$ Among these, phase 3 trials conducted in patients with CRSwNP uncontrolled with intranasal corticosteroids have demonstrated the efficacy of monoclonal antibody therapies targeting IL-4/IL-13 (dupilumab), IgE (omalizumab), and IL-5 (mepolizumab). ${ }^{58,63,64}$ The primary endpoints of these trials (reduction in nasal polyp score [NPS] and nasal congestion/obstruction score) were met with significant improvements in the treated patients versus placebo. Significant improvements in other measures including smell and disease-specific quality of life (SNOT-22) were also seen with all three drugs. Importantly, these phase 3 trials recruited a significant proportion of patients with comorbid asthma (48-71\%) and with prior surgery (58-100\%).

Dupilumab and omalizumab have received regulatory approval in the EU and US as add-on therapy for adult patients with severe CRSwNP inadequately controlled by intranasal corticosteroids, while mepolizumab is currently under regulatory review. The role of biologic therapy in the management of CRSwNP is currently evolving. Questions over the timing of biologic therapy versus surgery, the presence of comorbidities, and precise choice of biologic in individual patients remain to be determined, as highlighted in a recent publication by the European Forum for Research and Education in Allergy and Airway Diseases. ${ }^{65}$ Nevertheless, the availability of these targeted therapies is likely to lead to improvements in the management of patients with CRSwNP with unmet needs despite existing standard of care.

\section{Conclusion}

CRSwNP is a chronic predominantly type 2 inflammationmediated disease with an under-recognized clinical, humanistic, and economic burden despite the recalcitrant nature of the disease and the associated high symptom burden. Existing medical and surgical interventions, including intranasal corticosteroids and SCS and endoscopic sinus surgery, may be associated with recurrence and potential side effects and risks. As a result of the 
shared underlying type 2 inflammation implicated in the pathogenesis of several coexisting diseases, patients with CRSwNP often have comorbid asthma and/or NSAIDERD. These patients, as well as those with high corticosteroid use and/or sinonasal surgical history, have more severe disease and associated symptom burden and represent a difficult-to-treat population under the existing management paradigm. There exists an unmet need for effective and well-tolerated therapies for CRSwNP that effectively control symptoms, reduce NPS, improve HRQoL, and minimize relapse by targeting the underlying type 2 disease pathophysiology, thereby providing a holistic disease control. The judicious introduction of targeted biologic therapies into the treatment paradigm should lead to improvements in the lives of patients with this burdensome condition.

\section{Abbreviations}

COPD, chronic obstructive pulmonary disease; CRS, chronic rhinosinusitis; CRSwNP, chronic rhinosinusitis with nasal polyps; CT, computed tomography; HRQoL, health-related quality of life; IgE, immunoglobulin E; IL, interleukin; NSAID-ERD, nonsteroidal anti-inflammatory drug-exacerbated respiratory disease; SCS, systemic corticosteroid; SF-36, Short-Form Health Survey questionnaire.

\section{Acknowledgments}

We thank Siddhesh Kamat, of Regeneron Pharmaceuticals, Inc., for his contributions to the manuscript. Medical writing/editorial assistance was provided by Maggie Tarrio Watson, PhD, of Excerpta Medica, funded by Sanofi Genzyme and Regeneron Pharmaceuticals, Inc.

\section{Author Contributions}

All authors contributed to data analysis, drafting or revising the article, agreed on the journal to which the article was submitted, gave final approval of the version to be published, and agree to be accountable for all aspects of the work.

\section{Funding}

This work was supported by Sanofi and Regeneron Pharmaceuticals, Inc.

\section{Disclosure}

Professor Claus Bachert reports personal fees from and Principal Investigator of study and advisory board for GSK, Principal Investigator of study for AstraZeneca and Sanofi, consulting for Mylan, consulting and presentations for ALK, outside the submitted work; and is an advisory board member of ALK, AstraZeneca, GlaxoSmithKline, Intrexon Actobiotics, Novartis, Sanofi, and Stallergenes Greer.

Dr Neil Bhattacharyya reports personal fees from Sanofi, outside the submitted work and is a consultant for Sanofi.

Dr Martin Desrosiers reports personal fees from GlaxoSmithKline, grants from Sanofi and Regeneron, advisory board, speaker bureau, and clinical investigator for GlaxoSmithKline, Sanofi and Regeneron, and AstraZeneca, during the conduct of the study; is a major equity holder of Probionase Therapies outside the submitted work; has received clinical trial funding from AstraZeneca, GlaxoSmithKline, Probionase Therapies, and Sanofi; and is an advisory board member of Regeneron Pharmaceuticals, Inc. and Sanofi.

Dr Asif H Khan reports being an employee of Sanofi, during the conduct of the study and may hold stock and/or stock options.

\section{References}

1. Alobid I, Antón E, Armengot M, et al. SEAIC-SEORL. Consensus document on nasal polyposis. POLINA project. J Investig Allergol Clin Immunol. 2011;21(Suppl 1):1-58.

2. Hastan D, Fokkens WJ, Bachert C, et al. Chronic rhinosinusitis in Europe-an underestimated disease. A GA ${ }^{2}$ LEN study. Allergy. 2011;66(9):1216-1223. doi:10.1111/j.1398-9995.2011.02646.x

3. Stevens WW, Schleimer RP, Kern RC. Chronic rhinosinusitis with nasal polyps. J Allergy Clin Immunol Pract. 2016;4(4):565-572. doi:10.1016/j.jaip.2016.04.012

4. Bachert C, Pawankar R, Zhang L, et al. ICON: chronic rhinosinusitis. World Allergy Organ J. 2014;7(1):25. doi:10.1186/1939-4551-7-25

5. Bachert C, Mannent L, Naclerio RM, et al. Effect of subcutaneous dupilumab on nasal polyp burden in patients with chronic sinusitis and nasal polyposis: a randomized clinical trial. JAMA. 2016;315 (5):469-479. doi:10.1001/jama.2015.19330

6. Bachert C, Zhang N, Hellings PW, Bousquet J. Endotype-driven care pathways in patients with chronic rhinosinusitis. J Allergy Clin Immunol. 2018;141(5):1543-1551. doi:10.1016/j.jaci.2018.03.004

7. Fokkens WJ, Lund V, Bachert C, et al. EUFOREA consensus on biologics for CRSwNP with or without asthma. Allergy. 2019;74 (12):2312-2319. doi:10.1111/all.13875

8. Kim DW, Cho SH. Emerging endotypes of chronic rhinosinusitis and its application to precision medicine. Allergy Asthma Immunol Res. 2017;9(4):299-306. doi:10.4168/aair.2017.9.4.299

9. Khan A, Huynh TMT, Vandeplas G, et al. The GALEN rhinosinusitis cohort: chronic rhinosinusitis with nasal polyps affects health-related quality of life. Rhinology. 2019;57(5):343-351. doi:10.4193/ Rhin 19.158

10. van Agthoven M, Fokkens WJ, van de Merwe JP, Marijke van Bolhuis E, Uyl-de Groot CA, Busschbach JJ. Quality of life of patients with refractory chronic rhinosinusitis: effects of filgrastim treatment. Am J Rhinol. 2001;15(4):231-237. doi:10.1177/ 194589240101500403

11. Wensing M, Vingerhoets E, Grol R. Functional status, health problems, age and comorbidity in primary care patients. Qual Life Res. 2001;10(2):141-148. doi:10.1023/A:1016705615207 
12. Hosemann W, Draf C. Danger points, complications and medico-legal aspects in endoscopic sinus surgery. GMS Curr Top Otorhinolaryngol Head Neck Surg. 2013;12:Doc06. doi:10.3205/cto000098

13. Leung RM, Dinnie K, Smith TL. When do the risks of repeated courses of corticosteroids exceed the risks of surgery? Int Forum Allergy Rhinol. 2014;4(11):871-876. doi:10.1002/alr.21377

14. Vennik J, Eyles C, Thomas M, et al. Chronic rhinosinusitis: a qualitative study of patient views and experiences of current management in primary and secondary care. BMJ Open. 2019;9(4): e022644. doi:10.1136/bmjopen-2018-022644

15. Bhattacharyya N, Gilani S. Prevalence of potential adult chronic rhinosinusitis symptoms in the United States. Otolaryngol Head Neck Surg. 2018;159(3):522-525. doi:10.1177/0194599818774006

16. Hedman J, Kaprio J, Poussa T, Nieminen MM. Prevalence of asthma, aspirin intolerance, nasal polyposis and chronic obstructive pulmonary disease in a population-based study. Int J Epidemiol. 1999;28 (4):717-722. doi:10.1093/ije/28.4.717

17. Klossek JM, Neukirch F, Pribil C, et al. Prevalence of nasal polyposis in France: a cross-sectional, case-control study. Allergy. 2005;60 (2):233-237. doi:10.1111/j.1398-9995.2005.00688.x

18. Benjamin MR, Stevens WW, Li N, et al. Clinical characteristics of patients with chronic rhinosinusitis without nasal polyps in an academic setting. J Allergy Clin Immunol Pract. 2019;7(3):1010-1016. doi:10.1016/j.jaip.2018.10.014

19. Bachert C, Van Bruaene N, Toskala E, et al. Important research questions in allergy and related diseases: 3-chronic rhinosinusitis and nasal polyposis - a GALEN study. Allergy. 2009;64(4):520-533. doi:10.1111/ j.1398-9995.2009.01964.x

20. Fokkens WJ, Lund VJ, Hopkins C, et al. European position paper on rhinosinusitis and nasal polyps 2020. Rhinology. 2020;58(Suppl S29):1-464. doi:10.4193/Rhin20.401

21. Alobid I, Cardelus S, Benítez P, et al. Persistent asthma has an accumulative impact on the loss of smell in patients with nasal polyposis. Rhinology. 2011;49(5):519-524. doi:10.4193/Rhino10.295

22. Tomassen P, Vandeplas G, Van Zele T, et al. Inflammatory endotypes of chronic rhinosinusitis based on cluster analysis of biomarkers. J Allergy Clin Immunol. 2016;137(5):1449-1456.e1444. doi:10.1016/j.jaci.2015.12.1324

23. Bachert C, Gevaert P, Holtappels G, Johansson SG, van Cauwenberge P. Total and specific IgE in nasal polyps is related to local eosinophilic inflammation. J Allergy Clin Immunol. 2001;107 (4):607-614. doi:10.1067/mai.2001.112374

24. Chaaban MR, Walsh EM, Woodworth BA. Epidemiology and differential diagnosis of nasal polyps. Am J Rhinol Allergy. 2013;27 (6):473-478. doi:10.2500/ajra.2013.27.3981

25. Dietz de Loos DAE, Hopkins C, Fokkens WJ. Symptoms in chronic rhinosinusitis with and without nasal polyps. Laryngoscope. 2013;123(1):57-63. doi:10.1002/lary.23671

26. Bachert C, Claeys SE, Tomassen P, van Zele T, Zhang N. Rhinosinusitis and asthma: a link for asthma severity. Curr Allergy Asthma Rep. 2010;10(3):194-201. doi:10.1007/s11882-010-0096-0

27. Dykewicz MS, Rodrigues JM, Slavin RG. Allergic fungal rhinosinusitis. $J$ Allergy Clin Immunol. 2018;142(2):341-351. doi:10.1016/j.jaci.2018.06.023

28. Suh JD, Ramakrishnan V, Palmer JN. Biofilms. Otolaryngol Clin North Am. 2010;43(3):521-530, viii. doi:10.1016/j.otc.2010.02.010

29. Van Zele T, Gevaert P, Watelet JB, et al. Staphylococcus aureus colonization and $\mathrm{IgE}$ antibody formation to enterotoxins is increased in nasal polyposis. J Allergy Clin Immunol. 2004;114(4):981-983. doi:10.1016/j.jaci.2004.07.013

30. Mazzone SB, Undem BJ. Vagal afferent innervation of the airways in health and disease. Physiol Rev. 2016;96(3):975-1024. doi:10.1152/ physrev.00039.2015

31. Batra PS, Tong L, Citardi MJ. Analysis of comorbidities and objective parameters in refractory chronic rhinosinusitis. Laryngoscope. 2013;123(Suppl 7):S1-S11. doi:10.1002/lary.24418
32. Håkansson K, Thomsen SF, Konge L, Mortensen J, Backer V, von Buchwald C. A comparative and descriptive study of asthma in chronic rhinosinusitis with nasal polyps. Am J Rhinol Allergy. 2014;28(5):383-387. doi:10.2500/ajra.2014.28.4076

33. Mullol J, Picado C. Rhinosinusitis and nasal polyps in aspirin-exacerbated respiratory disease. Immunol Allergy Clin North Am. 2013;33(2):163-176. doi:10.1016/j.iac.2012.11.002

34. Stevens WW, Peters AT, Hirsch AG, et al. Clinical characteristics of patients with chronic rhinosinusitis with nasal polyps, asthma, and aspirin-exacerbated respiratory disease. J Allergy Clin Immunol Pract. 2017;5(4):1061-1070.e1063. doi:10.1016/j.jaip.2016.12.027

35. Bhattacharyya N, Villeneuve S, Joish VN, et al. Cost burden and resource utilization in patients with chronic rhinosinusitis and nasal polyps. Laryngoscope. 2019;129(9):1969-1975. doi:10.1002/lary.27852

36. Mendelsohn D, Jeremic G, Wright ED, Rotenberg BW. Revision rates after endoscopic sinus surgery: a recurrence analysis. Ann Otol Rhinol Laryngol. 2011;120(3):162-166. doi:10.1177/000348941112000304

37. Promsopa C, Kansara S, Citardi MJ, Fakhri S, Porter P, Luong A. Prevalence of confirmed asthma varies in chronic rhinosinusitis subtypes. Int Forum Allergy Rhinol. 2016;6(4):373-377. doi:10.1002/alr.21674

38. Bhan N, Kawachi I, Glymour MM, Subramanian SV. Time trends in racial and ethnic disparities in asthma prevalence in the United States from the Behavioral Risk Factor Surveillance System (BRFSS) study (1999-2011). Am J Public Health. 2015;105(6):1269-1275. doi:10.2105/AJPH.2014.302172

39. Heffler E, Blasi F, Latorre M, et al. The severe asthma network in Italy: findings and perspectives. J Allergy Clin Immunol Pract. 2019;7(5):1462-1468. doi:10.1016/j.jaip.2018.10.016

40. Khan A, Vandeplas G, Huynh TMT, et al. The global allergy and asthma European network (GALEN) rhinosinusitis cohort: a large European cross-sectional study of chronic rhinosinusitis patients with and without nasal polyps. Rhinology. 2019;57(1):32-42. doi:10.4193/ Rhin 17.255

41. Won HK, Kim YC, Kang MG, et al. Age-related prevalence of chronic rhinosinusitis and nasal polyps and their relationships with asthma onset. Ann Allergy Asthma Immunol. 2018;120(4):389-394. doi:10.1016/j.anai.2018.02.005

42. Laidlaw TM, Boyce JA, Longo DL. Aspirin-exacerbated respiratory disease-new prime suspects. $N$ Engl J Med. 2016;374(5):484-488. doi:10.1056/NEJMcibr1514013

43. Kowalski ML, Agache I, Bavbek S, et al. Diagnosis and management of NSAID-Exacerbated Respiratory Disease (N-ERD)-a EAACI position paper. Allergy. 2019;74(1):28-39. doi:10.1111/all.13599

44. Soler ZM, Wittenberg E, Schlosser RJ, Mace JC, Smith TL. Health state utility values in patients undergoing endoscopic sinus surgery. Laryngoscope. 2011;121(12):2672-2678. doi:10.1002/lary.21847

45. Gliklich RE, Metson R. The health impact of chronic sinusitis in patients seeking otolaryngologic care. Otolaryngol Head Neck Surg. 1995;113(1):104-109. doi:10.1016/S0194-5998(95)70152-4

46. Langdon C, Mullol J. Nasal polyps in patients with asthma: prevalence, impact, and management challenges. J Asthma Allergy. 2016;9:45-53. doi:10.2147/JAA.S86251

47. Castillo JA, Plaza V, Rodrigo G, Julia B, Mullol J. Chronic rhinosinusitis with and without nasal polyps and rhinitis in adult asthma. Frequency distribution and relationship with asthma control and severity (the IRIS-ASMA study). Eur Respir J. 2013;42(Suppl 57):3448.

48. Chung JH, Lee YJ, Kang TW, et al. Altered quality of life and psychological health (SCL-90-R) in patients with chronic rhinosinusitis with nasal polyps. Ann Otol Rhinol Laryngol. 2015;124 (8):663-670. doi:10.1177/0003489415576181

49. Erskine SE, Philpott CM. An unmet need: patients with smell and taste disorders. Clin Otolaryngol. 2020;45(2):197-203. doi:10.1111/ coa. 13484 
50. Värendh M, Johannisson A, Hrubos-Strøm H, Andersson M. Sleep quality improves with endoscopic sinus surgery in patients with chronic rhinosinusitis and nasal polyposis. Rhinology. 2017;55 (1):45-52. doi:10.4193/Rhin 16.065

51. Rudmik L, Smith TL, Schlosser RJ, Hwang PH, Mace JC, Soler ZM. Productivity costs in patients with refractory chronic rhinosinusitis. Laryngoscope. 2014;124(9):2007-2012. doi:10.1002/lary.24630

52. Lourijsen ES, Fokkens WJ, Reitsma S. Direct and indirect costs of adult patients with chronic rhinosinusitis with nasal polyps. Rhinology. 2020;58(3):213-217. doi:10.4193/Rhin19.468

53. Orlandi RR, Kingdom TT, Hwang PH, et al. International consensus statement on allergy and rhinology: rhinosinusitis. Int Forum Allergy Rhinol. 2016;6(Suppl 1):S22-S209. doi:10.1002/alr.21695

54. Hox V, Lourijsen E, Jordens A, et al. Benefits and harm of systemic steroids for short- and long-term use in rhinitis and rhinosinusitis: an EAACI position paper. Clin Transl Allergy. 2020;10:1. doi:10.1186/ s13601-019-0303-6

55. Waljee AK, Rogers MA, Lin P, et al. Short term use of oral corticosteroids and related harms among adults in the United States: population based cohort study. BMJ. 2017;357:j1415. doi:10.1136/ bmj.j1415

56. Smith KA, Orlandi RR, Oakley G, Meeks H, Curtin K, Alt JA. Longterm revision rates for endoscopic sinus surgery. Int Forum Allergy Rhinol. 2019;9(4):402-408. doi:10.1002/alr.22264

57. Calus L, Van Bruaene N, Bosteels C, et al. Twelve-year follow-up study after endoscopic sinus surgery in patients with chronic rhinosinusitis with nasal polyposis. Clin Transl Allergy. 2019;9:30. doi:10.1186/s13601-019-0269-4

58. Bachert C, Han JK, Desrosiers M, et al. Efficacy and safety of dupilumab in patients with severe chronic rhinosinusitis with nasal polyps (LIBERTY NP SINUS-24 and LIBERTY NP SINUS-52): results from two multicentre, randomised, double-blind, placebo-controlled, parallel-group phase 3 trials. Lancet. 2019;394 (10209):1638-1650. doi:10.1016/S0140-6736(19)31881-1
59. Bachert C, Sousa AR, Lund VJ, et al. Reduced need for surgery in severe nasal polyposis with mepolizumab: randomized trial. J Allergy Clin Immunol. 2017;140(4):1024-1031.e1014. doi:10.1016/j. jaci.2017.05.044

60. Bidder T, Sahota J, Rennie C, Lund VJ, Robinson DS, Kariyawasam HH. Omalizumab treats chronic rhinosinusitis with nasal polyps and asthma together-a real life study. Rhinology. 2018;56(1):42-45. doi:10.4193/Rhin17.139

61. Bachert C, Zhang N. Medical algorithm: diagnosis and treatment of chronic rhinosinusitis. Allergy. 2020;75(1):240-242. doi:10.1111/ all.13823

62. Clinicaltrials.gov. Efficacy and safety study of benralizumab in patient with eosinophilic chronic rhinosinusitis with nasal polyps (ORCHID); 2019. Available from: https://clinicaltrials.gov/ct2/ show/NCT04157335. Accessed August 12, 2020.

63. Gevaert P, Omachi TA, Corren J, et al. Efficacy and safety of omalizumab in nasal polyposis: 2 randomized phase 3 trials. $J$ Allergy Clin Immunol. 2020;146(3):595-605. doi:10.1016/j. jaci.2020.05.032

64. Hopkins C, Bachert C, Fokkens W, et al. Add-on mepolizumab for chronic rhinosinusitis with nasal polyps: SYNAPSE study. Eur Respir J. 2020;56(suppl 64):4616.

65. Bachert C, Han JK, Wagenmann M, et al. EUFOREA expert board meeting on uncontrolled severe Chronic Rhinosinusitis with Nasal Polyps (CRSwNP) and biologics: definitions and management. J Allergy Clin Immunol. 2020. doi:10.1016/j.jaci.2020.11.013

\section{Publish your work in this journal}

The Journal of Asthma and Allergy is an international, peer-reviewed open-access journal publishing original research, reports, editorials and commentaries on the following topics: Asthma; Pulmonary physiology; Asthma related clinical health; Clinical immunology and the immunological basis of disease; Pharmacological interventions and new therapies. The manuscript management system is completely online and includes a very quick and fair peer-review system, which is all easy to use. Visit http://www.dovepress.com/testimonials.php to read real quotes from published authors. 